\title{
Equivalência de Estímulos e Ciúme: Efeito de História Pré-Experimental
}

\section{Stimulus Equivalence and Jealousy: Effect of Pre-Experimental History}

\author{
Verônica Bender Haydu*, $a$, Larissa Botelho Gaça ${ }^{b}$, Natália Pascon Cognetti ${ }^{c}$, \\ Carlos Eduardo Costa ${ }^{a} \&$ Gerson Yukio Tomanari ${ }^{d}$ \\ ${ }^{a}$ Universidade Estadual de Londrina, Londrina, PR, Brasil, ${ }^{b}$ Universidade Federal de São Paulo, São Paulo, SP, Brasil, \\ ${ }^{c}$ Universidade Estadual de Maringá, Maringá, PR, Brasil \& ${ }^{d}$ Universidade de São Paulo, São Paulo, SP, Brasil
}

\begin{abstract}
Resumo
A formação de classes de estímulos equivalentes tem sido usada como modelo experimental para investigar uma série de fenômenos comportamentais, tais como o desenvolvimento de comportamento simbólico e a transferência de funções que os eventos ambientais adquirem por meio de relações condicionais. O objetivo deste estudo consistiu em analisar o efeito de estímulos com alto grau afetivo pré-experimentalmente constituído, na formação de classes de equivalência estabelecidas em contexto experimental, quando as relações emergentes podem se caracterizar por um conflito na escolha dos estímulos. Participaram 17 estudantes universitários que foram submetidos ao procedimento de escolha de acordo com modelo e aos testes das relações emergentes, para formar quatro classes de equivalência com quatro estímulos cada. Verificou-se que 10 dos 17 participantes formaram as classes equivalentes e observou-se correlação positiva entre o desempenho no Teste de Equivalência e o escore na Escala de Ciúme Romântico, sugerindo que a história pré-experimental referente ao envolvimento afetivo interferiu no desempenho dos participantes. $\mathrm{O}$ efeito de interferência provavelmente é devido ao conflito entre contingências: uma que controla o responder diante de relações condicionais pré-experimentalmente estabelecidas, envolvendo estímulos com alto valor afetivo, e outra estabelecida no contexto experimental.

Palavras-chave: Classes de equivalência, transferência de funções, ciúme romântico, escolha de acordo com modelo.

Abstract

The formation of stimulus equivalence classes has been used as an experimental model to investigate a series of behavioral phenomena, such as the development of symbolic behavior and transfer of functions that environmental events acquire through conditional relations. The aim of this study was to analyze the effect of stimuli with high pre-experimentally constituted affective grade in the formation of equivalence classes established in an experimental context, when the emergent relations can be characterized by a conflict in choosing stimuli. Participants were 17 college students exposed to matching to sample procedure and the tests of emergent relations to form four equivalence classes with four stimuli each. The results showed that 10 out of 17 participants formed equivalence classes, and positive correlation was observed between the performance on the Equivalence Test and the score on the Romantic Jealousy Scale, suggesting that the pre-experimental history related to emotional involvement interfered with participants' performance. The interference effect is probably due to the conflict between contingencies: one that controls responses to pre-experimentally established conditional relations involving stimuli with high emotional value, and another one, established in the experimental setting.

Keywords: Equivalence classes, transfer of function, romantic jealousy, matching to sample.
\end{abstract}

Um grande número de pesquisas sobre formação de classes de estímulos equivalentes foi iniciado a partir do estudo desenvolvido por Sidman (1971) e das formula- ções teóricas apresentadas posteriormente (Sidman, 1986; Sidman \& Tailby, 1982). Esse processo comportamental é demonstrado quando se ensinam duas ou mais rela- 
Haydu, V. B., Gaça, L. B., Cognetti, N. P., Costa, C. E. \& Tomanari, G. Y. (2015). Equivalência de Estímulos e Ciúme: Efeito de História Pré-Experimental.

ções condicionais entre estímulos com um elemento em comum e emergem relações que não foram diretamente ensinadas. No ensino, geralmente, é empregado o procedimento de escolha de acordo com modelo (matching to sample - MTS), de tal forma que são treinadas relações condicionais entre, por exemplo, A1 e B1, B1 e C1, A2 e B2, B2 e C2 (essas siglas alfanuméricas são usadas sem a conjunção "e" para representar as relações entre estímulos treinadas ou testadas). Para demonstrar a formação de classes de estímulos equivalentes são realizados testes de simetria (B1A1, B2A2, C1B1, C2B2), de reflexividade (B1B1, C1C1, A2A2, B2B2, C2C2), de transitividade (A1C1, A2C2) e de transitividade simétrica $(\mathrm{C} 1 \mathrm{~A} 1$, C2A2). Uma vez que as classes tenham sido formadas, verifica-se que os estímulos tornam-se substituíveis entre si, apesar de não serem idênticos fisicamente (Sidman, 1986, 2000).

Na maioria das pesquisas que surgiu a partir da década de 1970, foi testado o efeito de diversas variáveis sobre o processo de formação das classes de equivalência (ver Barros, Galvão, Brino, Goulart, \& Mcllvane, 2005 e Sidman, 1994, para revisão), tendo-se identificado que, dentre outras, as seguintes variáveis afetam a probabilidade de as classes emergirem: as características dos estímulos e a possibilidade de eles serem nomeados (e.g., Arntzen \& Torunn, 2010; Medeiros \& Nogueira, 2005); o número de estímulos relacionados nas classes de equivalência e o número de classes (e.g., Arntzen \& Holth, 2000; Sidman, Kirk, \& Willson-Morris, 1985); a estrutura de treino, a distância nodal entre os estímulos das relações condicionais e o tipo de protocolo de treino e de teste (e.g., Imam, 2006; Moss-Lourenco \& Fields, 2011; Saunders \& Green, 1999); a topografia de controle de estímulos e a topografia das repostas (e.g., Dube \& McIlvane, 1996; Kato, de Rose, \& Faleiros, 2008); a história experimental e pré-experimental dos participantes (e.g., Fields, Arntzen, Nartey, \& Eilifsen, 2012; Neves et al., 1999).

Com relação ao efeito da história experimental, os estudos têm adotado dois tipos de procedimento: (a) uma função de estímulo, por exemplo, eliciadora, discriminativa, reforçadora, é condicionada após as classes de equivalência terem sido formadas e, em seguida, testa-se o efeito de transferência da função condicionada (e.g., Dymond, Roche, Forsyth, Whelan, \& Rhoden, 2008); (b) uma função de estímulo é condicionada antes de serem treinadas as relações condicionais que possibilitarão a formação das classes e nos testes é avaliado se os demais membros da classe adquiriram essa função (e.g., Tyndall, Roche, \& James, 2004). Quanto ao efeito de história pré-experimental, aspecto de interesse do presente estudo, verificou-se que funções de estímulos de diversos tipos podem bloquear ou interferir na formação de classes de equivalência, por envolverem controle de estímulos conflituoso com as classes estabelecidas pelo experimentador ou envolverem estímulos que provocam respostas de ansiedade, esquiva etc. Esse tipo de efeito foi estudado, por exemplo, por
Dixon, Refeldt, Zlomke e Robinson (2006); Fields et al. (2012); Leslie, Ulster-Jordanstown, Tierney, Robinson e Keenan (1993); Neves et al. (1999); Plaud (1995); Plaud, Gaither, Franklin, Weller e Barth (1998), Watt, Keenan, Barnes e Cairns (1991).

$\mathrm{O}$ estabelecimento de relações com controle de estímulos conflituosos, envolvendo estímulos que eliciam respostas de ansiedade, foi realizado no estudo de Leslie et al. (1993), do qual participaram oito pacientes diagnosticados como ansiosos que faziam psicoterapia e oito não ansiosos. Os estímulos geradores de ansiedade eram as seguintes expressões: EXAMS (A1), JOB INTERVIEW (A2), e PUBLIC SPEAKING (A3). Esses estímulos foram relacionados por meio do procedimento de MTS a sílabas sem sentido: ZID (B1), VEK (B2) e YIM (B3). Esses, por sua vez, foram relacionados a palavras que se referem a situações prazerosas: FULFILLED (C1), RELAXED (C2) e COMFORTABLE (C3). Todos os participantes responderam de acordo com as contingências estabelecidas pelos experimentadores nos testes de linha de base e seis dos oito participantes não ansiosos demonstraram as relações emergentes CA. Entretanto, apenas um dos oito participantes ansiosos demonstrou formação das classes de equivalência. Os autores concluíram que a ansiedade clinicamente relevante interfere com a formação de classes de equivalência que envolvem expressões de situações que geram ansiedade com expressões de prazer, conforto e relaxamento.

Estímulos que eliciam respostas de ansiedade foram empregados, também, no estudo de Plaud (1995), em que foi comparado o efeito da inclusão nas classes de estímulos temidos pelas participantes (nomes de espécies de cobras) e de estímulos que não eliciavam respostas de medo (nomes de flores). O procedimento consistiu de treino de relações condicionais para formar dois conjuntos de classes de estímulos equivalentes, sendo um com os nomes de flores e o outro com os nomes de cobras. Os resultados revelaram uma interação significativa entre a formação de classes equivalentes e o tipo de estímulo. Quando eram nomes de cobras, as participantes que relataram ter medo de cobra em um questionário aplicado na última fase do procedimento necessitaram de um maior número de blocos de treino e de testes para atingir $100 \%$ de acertos do que as participantes que relataram não ter medo de cobras. Resultados semelhantes foram obtidos por Plaud et al. (1998), em que foram usados como estímulos palavras com conotação sexual.

O resultado das pesquisas de Leslie et al. (1993), Plaud (1995) e Plaud et al. (1998), assim como outros citados anteriormente, permitem afirmar que o modelo de equivalência de estímulos é apropriado para investigar o efeito de relações pré-experimentais na formação de novas relações arbitrárias entre estímulos, possibilitando investigar, por meio de procedimentos de análise experimental do comportamento, temas muitas vezes difíceis de serem analisados em situação de laboratório, como o de atitudes 
(Grey \& Barnes, 1996), de preconceito (de Carvalho, 2009; Dixon et al., 2006), de abuso sexual (McGlinchey, Fairhurst, \& Dillenburger, 2000), de auto avaliação de eficácia (Dack, McHugh, \& Reed, 2012), entre outros. Um desses tópicos, o qual será objeto do presente estudo e que foi selecionado para testar essa hipótese, é o ciúme presente nas relações afetivas. $\mathrm{O}$ ciúme é um tema bastante polêmico, pois ao mesmo tempo em que é considerado um sentimento (Almeida, Rodrigues, \& Silva, 2008) é também tratado como sendo uma emoção (Banaco, 2005) ou como um comportamento (Costa \& Barros, 2010; Skinner, 1948/1976). Para Almeida et al., ele é um sentimento importante por marcar a existência de compromisso entre parceiros, mas também é considerado como um sentimento que prejudica os relacionamentos. Segundo Costa e Barros (2010), o ciúme é um comportamento complexo formado por um conjunto de operantes e de respondentes interconectados, e funcionalmente relacionados a eventos ambientais. Esses autores sugerem tratar-se de um comportamento estabelecido, principalmente, pela competição por reforços e mantido por reforço negativo, como a remoção do rival e/ou atenuação da competição.

A importância de se investigar processos que podem modificar ou impedir o estabelecimento de relações de estímulos equivalentes levou ao questionamento de se a função afetiva/emocional pré-experimental do nome do parceiro romântico impede ou bloqueia a formação de relações condicionais que possam apresentar conflito, impedindo a formação de classes de equivalência. Estudos dessa natureza são relevantes tanto com respeito à possibilidade de se esclarecer as variáveis relevantes envolvidas nesse processo quanto ao aspecto da aplicação desse conhecimento a contextos clínicos em que se visa alterar a função de estímulos que causam sofrimento ou prejuízos ao cliente. Assim, o objetivo do presente estudo consistiu em analisar o efeito de estímulos com alto grau afetivo pré-experimentalmente constituído, na formação de classes de equivalência estabelecidas em contexto experimental, quando as relações emergentes podem se caracterizar por um conflito no controle de estímulos. Como estímulos com alto grau afetivo pré-experimentalmente foi selecionado, dentre outros, um que provavelmente envolve ciúme, por ser esse um comportamento ainda pouco explorado em estudos de Análise do Comportamento (Costa \& Barros, 2010) e devido a sua relevância do ponto de vista psicológico. O ciúme se caracteriza como tendo valor adaptativo, mas também podendo vir a ser um distúrbio de comportamento (Almeida et al., 2008). Tal como no estudo de Plaud (1995), aplicou-se um instrumento no final do procedimento em que os participantes relataram verbalmente como se sentiam, o que no presente estudo foi relacionado ao sentimento de ciúme em relação ao parceiro romântico.

\section{Método}

\section{Participantes}

Dezessete estudantes universitários (16 do sexo feminino e um do sexo masculino) que cursavam o $1^{\circ}$ ano do curso de Psicologia de uma instituição pública, com idades entre 17 e 22 anos participaram da pesquisa. $O$ critério de seleção dos participantes consistiu em estarem namorando há pelo menos 6 meses ininterruptamente. A diferença no número de participantes em relação ao gênero se deve ao fato de haver uma proporção diferente de alunos de Psicologia no que se refere esse aspecto.

\section{Local e Materiais}

A coleta de dados foi realizada em uma sala de um laboratório de Análise Experimental do Comportamento Humano, contendo uma mesa e uma cadeira e, sobre a mesa, um microcomputador, com o Software Equivalência (Capocio, 2008) instalado. Esse software foi usado para a coleta de dados, assim como um formulário para levantamento dos dados pessoais dos participantes, uma folha de papel com uma lista de nomes próprios, e a Escala de Ciúme Romântico validada por Ramos, Yazawa e Salazar(1994). Essa foi a única escala validada para a população brasileira disponível no período da coleta de dados do presente estudo, para a qual foram obtidos os critérios para o estabelecimento dos escores de ciúme (os autores do presente estudo agradecem ao Prof. Dr. André Ramos pelo fornecimento dessas informações).

A Escala de Ciúme Romântico apresenta uma lista de frases que especificam situações de relacionamento, por exemplo: "Trocar o meu nome pode ser uma questão de esquecimento"; "Acho ruim quando ele fala que já teve momentos muito bons com outra pessoa". A tarefa do participante consiste em indicar, para cada uma das situações, a sua opinião a respeito de possíveis comportamentos de seu companheiro, colocando num parênteses ao lado da frase o número que melhor representa sua opinião: (1) Discordo completamente; (2) Discordo; (3) Em dúvida; (4) Concordo; (5) Concordo plenamente. Para o presente estudo foram selecionadas 20 das 32 sentenças que eram mais apropriadas para o contexto atual da pesquisa aqui relatada e a faixa etária dos participantes, como ambientes frequentados por jovens e o tipo de situação de interação mais frequente em suas vidas, sendo que foram mantidas as de número: $2,3,5,7,8,12,13,15,16,18,19,20,22$, $24,25,27,28,30,31,32$.

Os estímulos não familiares (desenhos) e o tipo de nome utilizado para o estabelecimento de relações condicionais para os participantes do gênero masculino e do feminino estão representados na Tabela 1 . O nome do(a) namorado(a) de cada participante foi fornecido no preenchimento do formulário de levantamento de dados pessoais. $\mathrm{O}$ conjunto de estímulos formados por nomes de pessoas desconhecidas foi composto a partir dos que cada participante selecionou na lista de nomes próprios a ele fornecido. 
Haydu, V. B., Gaça, L. B., Cognetti, N. P., Costa, C. E. \& Tomanari, G. Y. (2015). Equivalência de Estímulos e Ciúme: Efeito de História Pré-Experimental.

Tabela 1

Estímulos Apresentados aos Participantes de Ambos os Gêneros como Estímulos-Modelo ou de Comparação. Os Números Indicam as Classes de Estímulos a Serem Formadas e as Letras o Conjunto de Estímulos que Compõem as Classes

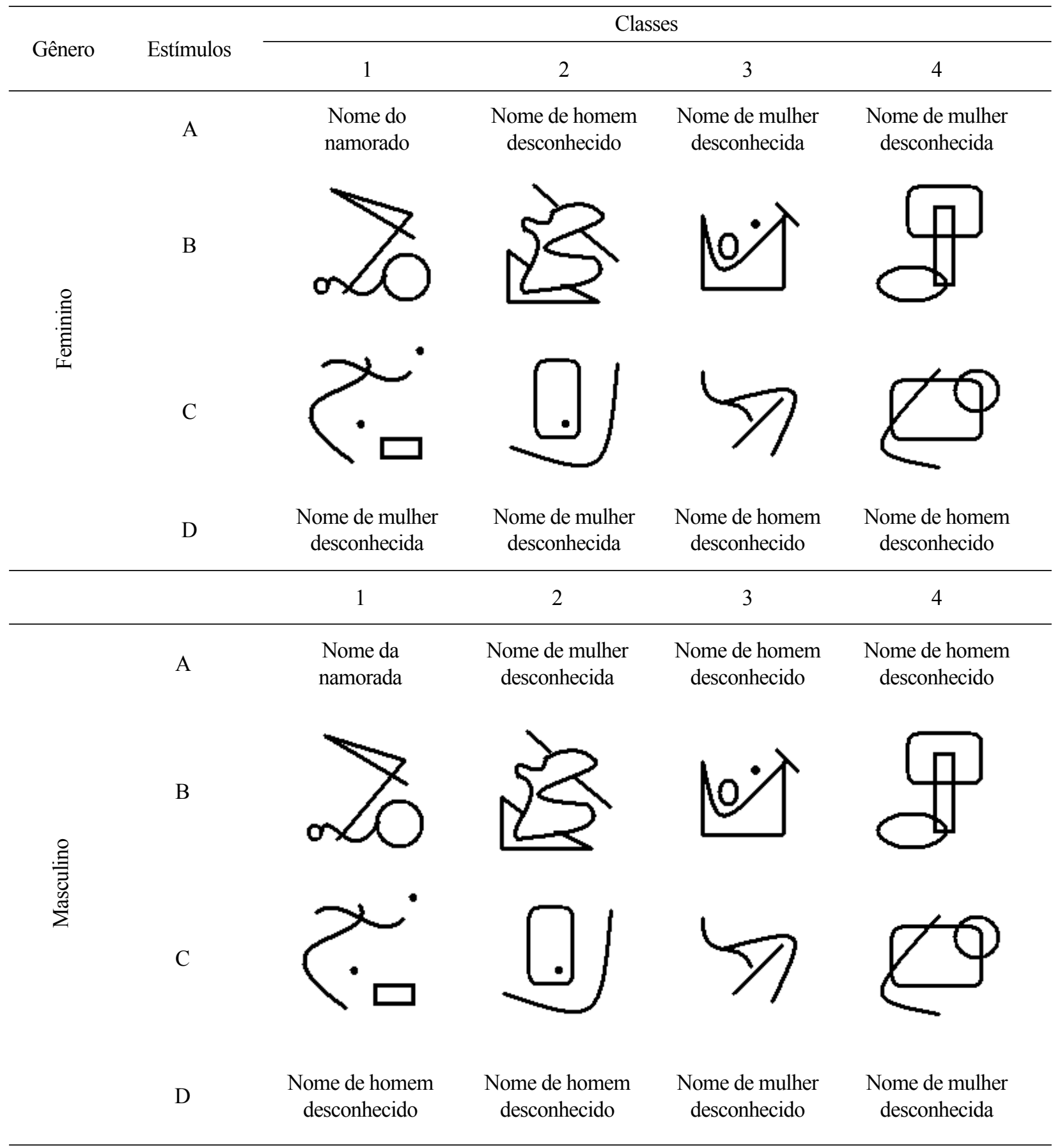

\section{Procedimento}

Os alunos interessados em participar foram convidados a comparecerem ao laboratório para a apresentação da pesquisa, onde foram esclarecidos sobre como era o procedimento e solicitava-se a leitura e assinatura do Termo de Consentimento Livre e Esclarecido (aprovado pelo comitê de ética em pesquisa da instituição dos autores) caso concordassem em participar da pesquisa. Ainda nesse encontro, após a assinatura do Termo, eles responderam a um questionário de dados pessoais, sendo que uma das questões solicitava o nome do namorado (ou namorada) do participante. Em seguida, a lista de nomes próprios foi entregue, para que eles selecionassem nomes que não correspondiam aos de pessoas conhecidas por eles. O protocolo de treino e teste das relações entre estímulos foi personalizado para cada participante a partir das informações fornecidas pelos participantes nesse levantamento inicial. 
Os procedimentos de treino e o de teste foram iguais para os participantes de ambos os gêneros, exceto quanto aos estímulos "nome do namorado(a)" e "nome de pessoas desconhecidas". Antes de iniciar a sessão, as seguintes instruções eram lidas para todos os participantes:

Sua tarefa nesse experimento é relacionar estímulos que aparecerão na tela do computador através do clicar com o mouse sobre os estímulos. É de extrema importância que durante toda a fase de coleta de dados você não comente com ninguém sobre o procedimento da pesquisa ou sobre os estímulos que você verá. Não anote esses estímulos de nenhuma forma. O estímulo que aparecerá no centro da tela é denominado estímulo-modelo. Os quatro estímulos que aparecerem em volta dele são chamados estímulos de comparação. Você deve, então, clicar sobre o estímulo de comparação que você considere estar relacionado com aquele estímulo-modelo que está no centro da tela. Somente um estímulo de comparação pode ser relacionado com cada estímulo-modelo e você deverá descobrir quais são os pares corretos. Você passará por algumas fases e só avançará quando atingir um determinado critério de acertos. Ao término do experimento, se você quiser, poderá obter maiores informações sobre os resultados, mas durante o procedimento, nenhuma informação adicional poderá ser obtida.

$\mathrm{O}$ arranjo de treino era do tipo linear. As relações condicionais $\mathrm{A} 1 \mathrm{~B} 1, \mathrm{~B} 1 \mathrm{C} 1, \mathrm{C} 1 \mathrm{D} 1, \mathrm{~A} 2 \mathrm{~B} 2, \mathrm{~B} 2 \mathrm{C} 2, \mathrm{C} 2 \mathrm{D} 2$, $\mathrm{A} 3 \mathrm{~B} 3, \mathrm{~B} 3 \mathrm{C} 3, \mathrm{C} 3 \mathrm{D} 3, \mathrm{~A} 4 \mathrm{~B} 4, \mathrm{~B} 4 \mathrm{C} 4, \mathrm{C} 4 \mathrm{D} 4$ foram treinadas por meio do procedimento de MTS. O estímulo-modelo era apresentado no centro da tela do monitor e os estímulos de comparação em volta dele, distribuídos de forma equidistante. $O$ treino de relações entre estímulos foi misto, com todas as relações treinadas em um mesmo bloco. A Tabela 2 apresenta a sequência de treinos e testes do procedimento, as relações treinadas e/ou testadas em cada etapa e o número de tentativas nos blocos.

Tabela 2

Sequência de Treinos e Testes, Relações Treinadas ou Testadas em Cada Etapa e o Número de Tentativas em Cada Bloco

\begin{tabular}{ccc}
\hline $\begin{array}{c}\text { Etapas do } \\
\text { Procedimento }\end{array}$ & Relações & $\begin{array}{c}\text { Número de } \\
\text { Tentativas por Bloco }\end{array}$ \\
\hline Treino Misto & A1B1, B1C1, C1D1, A2B2, B2C2, C2D2, A3B3, B3C3, & 48 \\
& C3D3, A4B4, B4C4, C4D4 & \\
Teste Linha de Base & A1B1, B1C1, C1D1, A2B2, B2C2, C2D2, A3B3, B3C3, & 24 \\
& C3D3, A4B4, B4C4, C4D4 & \\
Teste Simetria & B1A1, C1B1, D1C1, B2A2, C2B2, D2C2, B3A3, C3B3, & 24 \\
& D3C3, B4A4, C4B4, D4C4 & \\
Teste de Transitividade e & A1C1, A1D1, B1D1, C1A1, D1A1, D1B1, A2C2, A2D2, & \\
Transitividade Simétrica & B2D2, C2A2, D2A2, D2B2, A3C3, A3D3, B3D3, C3A3, \\
& D3A3, D3B3, A4C4, A4D4, B4D4, C4A4, D4A4, D4B4 & 24 \\
\hline
\end{tabular}

$\mathrm{Na}$ etapa Treino Misto, foram ensinadas, por meio de MTS, as relações condicionais do tipo $\mathrm{AB}, \mathrm{BC}$ e $\mathrm{CD}$ em um bloco de 48 tentativas. Em cada tentativa havia um estímulo de comparação positivo, ou seja, correto de acordo com o critério especificado pelos experimentadores e três estímulos de comparação negativos, que eram os estímulos incorretos. As instruções dadas ao participante assim que ele estava para iniciar o primeiro bloco de treino eram:

As relações corretas entre o estímulo-modelo e o estímulo de comparação não são baseadas em semelhança física entre eles, sequência ou posição na tela. Portanto, você deverá descobrir como eles se relacionam através das consequências das escolhas que você fizer. Todas as suas escolhas serão seguidas por uma mensagem que indicará se elas foram corretas ou se precisam ser modificadas.

Todas as respostas dos participantes eram consequenciadas, informando o acerto ou erro. O critério exigido para a passagem para etapa seguinte era de $90 \%$ de acertos. Quando o participante não atingia esse critério, o bloco era repetido até que ele fosse atingido ou até o limite de 1 hora de sessão. Se esse critério de manutenção não fosse atingido dentro de $1 \mathrm{~h}$, o participante era informado de que a participação dele na pesquisa estava encerrada e os dados dele não eram tabulados.

No início da fase de testes a seguinte instrução foi lida:

A partir de agora você não receberá mais nenhuma mensagem sobre o seu desempenho, indicando acerto ou erro. Mesmo assim, continue respondendo de acordo com o que você aprendeu.

Na etapa Teste Linha de Base, eram testadas as relações condicionais do tipo AB, BC, CD. Na etapa Teste de Simetria, eram testadas as relações emergentes do tipo $\mathrm{BA}, \mathrm{CB}$, DC. O Teste de Transitividade e Transitividade Simétrica envolvia testar as relações emergentes do tipo $\mathrm{AC}, \mathrm{AD}$, BD, CA, DA, DB. Cada bloco de teste era composto por 24 tentativas. Os blocos de teste não eram repetidos qualquer que fosse o desempenho do participante. $\mathrm{O}$ treino $\mathrm{e}$ os testes eram realizados em uma única sessão para cada participante. Depois de passar por esse procedimento, 
Haydu, V. B., Gaça, L. B., Cognetti, N. P., Costa, C. E. \& Tomanari, G. Y. (2015). Equivalência de Estímulos e Ciúme: Efeito de História Pré-Experimental.

solicitava-se aos participantes que respondessem à Escala de Ciúme Romântico na mesma mesa em que estava o computador (Ramos et al., 1994).

\section{Resultados}

Todos os participantes concluíram as fases e etapas do procedimento. Para a análise de dados, considerou-se o escore obtido pelos participantes na Escala de Ciúme Romântico. De acordo com Ramos et al. (1994), o grau de ciúme é considerado Baixo quando o escore obtido pelo participante é acima de 3,5; Moderado, entre 3,4 e 2,05; Intenso, inferior a 2,04. Os resultados da classificação dos dados dos participantes encontram-se na $3^{\mathrm{a}}$ coluna da Tabela 3 ; e na $4^{\mathrm{a}}$ coluna, os escores obtidos por eles. $\mathrm{Na} 5^{\mathrm{a}}$ coluna, estão as porcentagens de respostas que estavam de acordo com as classes especificadas pelos pesquisadores (acertos) no conjunto dos três testes (Teste de Linha de Base; Teste de Simetria; Teste de Transitividade e de Transitividade Simétrica) denominado Teste de Equivalência Global, com 72 tentativas. $\mathrm{Na} 6^{\mathrm{a}}$ coluna da Tabela 3, estão as porcentagens de acertos diante das relações condicionais A1D1 e D1A1, do Teste de Transitividade e de Transitividade Simétrica. O estímulo A1 era o nome do namorado(a) e o estímulo D1 era o nome de um desconhecido(a). Esses dados foram comparados com os escores da Escala de Ciúme, tendo-se verificado, por meio da correlação de Spearman, que eles estão positivamente correlacionados $(\rho=0,5009, p<0,05)$. $\mathrm{Ou}$ seja, participantes que obtiveram escores mais altos na Escala de Ciúme (indicador de menor grau de ciúme) apresentaram porcentagens de acertos mais altas no Teste de Equivalência que envolvia o nome do namorado(a) e de uma pessoa do sexo oposto desconhecida.

O participante que revelou, por meio de relato verbal (Escala de Ciúme), ter ciúme em baixa intensidade, apresentou $100 \%$ de acertos no Teste de Equivalência. Dos cinco participantes que são classificados como tendo ciúme intenso, dois deles (P15 e P16) apresentaram as menores porcentagens de acertos nesse teste em comparação aos demais participantes e dois deles (P13 e P14) apresentaram porcentagens superiores à maioria dos participantes que relatou ciúme moderado. As porcentagens de acerto diante das relações condicionais A1D1 e D1A1, que envolviam o nome do namorado(a), também estão positivamente correlacionadas com os escores obtidos pelos participantes na Escala de Ciúme, de acordo com o coeficiente de correlação de Spearman $(\rho=0,6495, p<0,05)$. Quatro participantes (P12, P15, P16, P17) não emitiram respostas corretas na formação dessas relações, sendo três deles com ciúme intenso e um com ciúme moderado.

Tabela 3

Classificação e Escore na Escala de Ciúme Romântico, Porcentagens de Acertos no Teste de Equivalência Global, e no Teste das Relações A1-D1 e D1-A1 que Envolviam o Nome do Namorado(a)

\begin{tabular}{|c|c|c|c|c|c|}
\hline \multirow[t]{2}{*}{ Part. } & \multirow[t]{2}{*}{ Gênero } & \multicolumn{2}{|c|}{ Escala de Ciúme } & $\begin{array}{c}\text { Teste de Equivalência } \\
\text { Global }\end{array}$ & $\begin{array}{c}\text { Teste das Relações } \\
\text { A1-D1 e D1-A1 }\end{array}$ \\
\hline & & Classificação & Escore & Porcenta & ertos \\
\hline 1 & M & Baixo & 4,02 & 100 & 100 \\
\hline 2 & $\mathrm{~F}$ & Moderado & 3,2 & 100 & 100 \\
\hline 3 & $\mathrm{~F}$ & Moderado & 2,97 & 94,4 & 100 \\
\hline 4 & $\mathrm{~F}$ & Moderado & 2,92 & 94,4 & 100 \\
\hline 5 & $\mathrm{~F}$ & Moderado & 2,92 & 95,8 & 100 \\
\hline 6 & $\mathrm{~F}$ & Moderado & 2,8 & 100 & 100 \\
\hline 7 & $\mathrm{~F}$ & Moderado & 2,8 & 94,4 & 100 \\
\hline 8 & $\mathrm{~F}$ & Moderado & 2,8 & 86,1 & 100 \\
\hline 9 & $\mathrm{~F}$ & Moderado & 2,45 & 86,1 & 66,6 \\
\hline 10 & $\mathrm{~F}$ & Moderado & 2,45 & 66,6 & 100 \\
\hline 11 & $\mathrm{~F}$ & Moderado & 2,42 & 98,6 & 100 \\
\hline 12 & $\mathrm{~F}$ & Moderado & 2,12 & 72,2 & 0 \\
\hline 13 & $\mathrm{~F}$ & Intenso & 2,02 & 100 & 100 \\
\hline 14 & $\mathrm{~F}$ & Intenso & 2,02 & 97,2 & 100 \\
\hline 15 & $\mathrm{~F}$ & Intenso & 2,02 & 61,1 & 0 \\
\hline 16 & $\mathrm{~F}$ & Intenso & 1,7 & 55,5 & 0 \\
\hline 17 & $\mathrm{~F}$ & Intenso & 1,5 & 79,1 & 0 \\
\hline
\end{tabular}


Na Figura 1 estão os dados referentes às porcentagens de respostas que não estavam de acordo com as classes estabelecidas pelos experimentadores (erros) nos testes das relações de linha de base e das relações emergentes das Classes 1, 2, 3 e 4. Verifica-se nessa figura, que nos testes de linha de base e de simetria, as relações com maior porcentagem de erros são as relações $\mathrm{BC}$ e $\mathrm{CB}$, exceto no caso da Classe 4 em que não ocorreram erros de relações BC. Quanto aos testes de transitividade e transitividade simétrica, observa-se que as relações AD e DA da Classe 1 , que envolvia o nome do namorado(a) foram as mais erradas em comparação às relações $\mathrm{AC}$ e BD, CA e DB. Essa diferença no desempenho mostra uma tendência de haver mais erros na emergência da relação $\mathrm{AD}$ do que $\mathrm{AC}$ e maior porcentagem de erros $\mathrm{AC}$ do que $\mathrm{BD}$; assim também maior porcentagem de erros DA do que CA, e de $\mathrm{AC}$ maior do que de DB. A diferença entre a porcentagem de erros da relação AC e AD é de 14,3\%, e a diferença na porcentagem de erros da relação CA e DA é de 14,2\%. Esse grau de diferença não é observada em relação aos dados de nenhuma das demais classes, verificando-se inclusive que as relações $\mathrm{AD}$ (transitividade) da Classe 3 foram menos erradas do que as relações $\mathrm{AC}$ e $\mathrm{BD}$, e as relações DA (transitividade simétrica) da Classe 2 e 3 foram menos erradas do que as relações DB e DA, com diferenças de até $39,1 \%$ ao se comparar a porcentagem de erros da relação DA com DB da Classe 2.

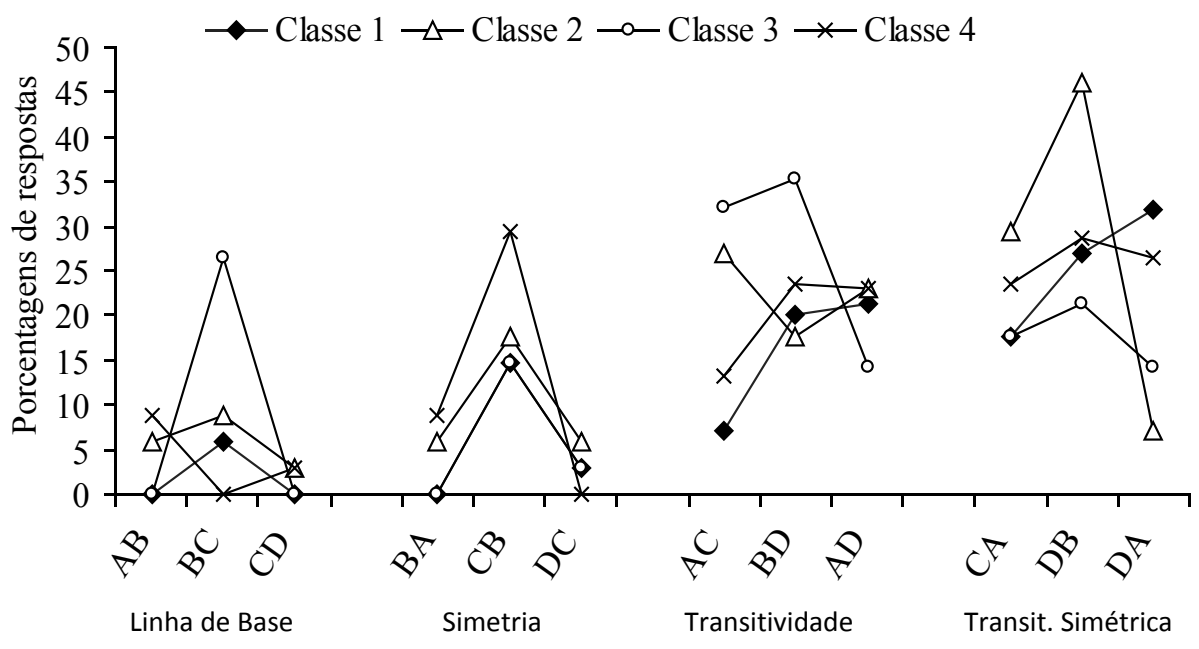

Relações condicionais

Figura 1. Porcentagens médias de respostas incorretas nos testes das relações condicionais emergentes de linha de base $(\mathrm{AB}, \mathrm{BC}$ e $\mathrm{CD})$, simetria $(\mathrm{BA}, \mathrm{CB}, \mathrm{DC})$, transitividade $(\mathrm{AC}, \mathrm{BD}$, $\mathrm{AD})$ e transitividade simétrica (CA, DB, DA) das classes 1, 2, 3 e 4.

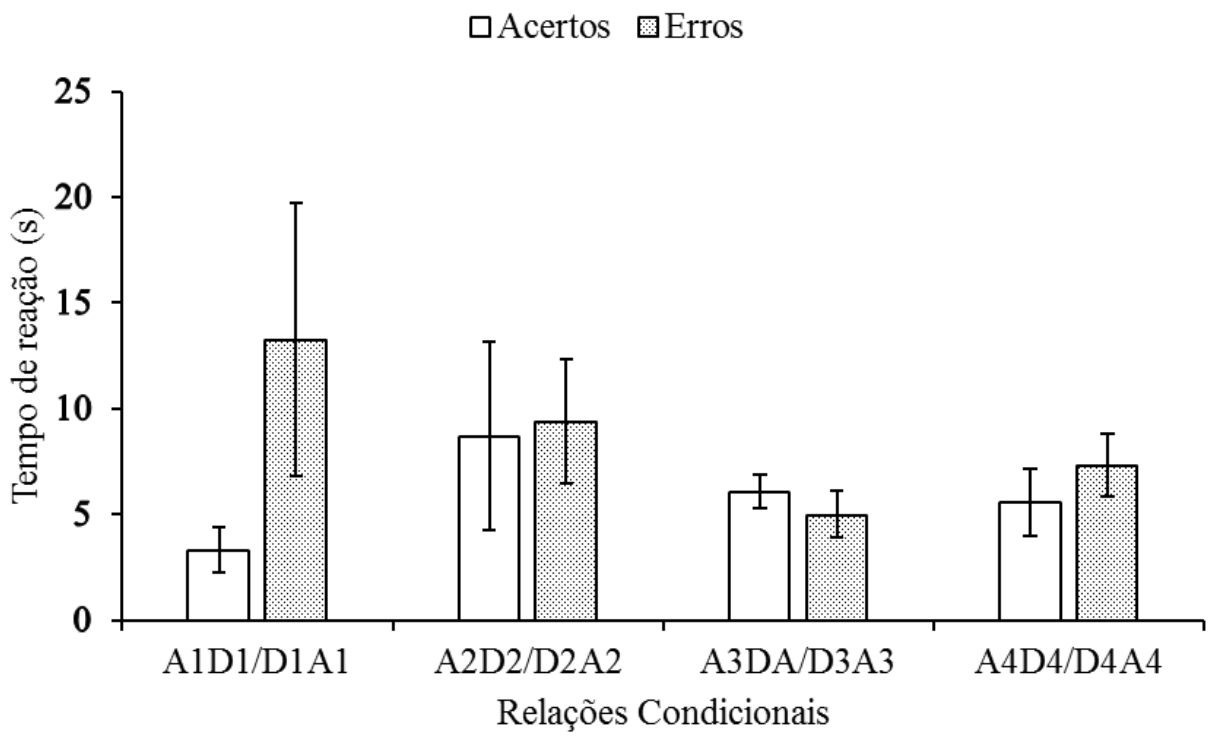

Figura 2. Tempo de reação médio em segundos nas tentativas com acertos e com erros diante das relações emergentes de transitividade (A1D1, A2D2, A3D3, A4D4) e transitividade simétrica (D1A1, D2A2, D3A3, D4A4) das classes 1, 2, 3 e 4. 
Haydu, V. B., Gaça, L. B., Cognetti, N. P., Costa, C. E. \& Tomanari, G. Y. (2015). Equivalência de Estímulos e Ciúme: Efeito de História Pré-Experimental.

Os tempos de reação médios em segundos nas tentativas em que as respostas estavam corretas e aquelas em que estavam incorretas foram comparados tomando como referência os dados das quatro classes em separado. Esses dados encontram-se na Figura 2, onde se observa que o tempo de reação é maior em tentativas com resposta incorreta do que naquelas com resposta correta, exceto no caso das relações das classes A3-D3/ D3-A3. Além disso, verifica-se que a maior diferença entre os tempos de reação, ao se considerar as tentativas com acertos e com erros, foi diante das relações da Classe 1 (9,94 s), que envolviam o nome do namorado(a) (A1D1 e D1A1), em comparação com as demais em que a diferença é de $1,85 \mathrm{~s}$ no máximo (A2D2 e D2A2).

\section{Discussão}

Dez dos 17 participantes formaram as classes de equivalência com base no critério de $90 \%$ de acertos nos testes das relações emergentes. Os dados obtidos sugerem que, no caso dos Participantes 15, 16 e 17, classificados como tendo ciúme intenso, e dos Participantes 8, 9, 10 e 12, classificados como tendo ciúme moderado, a história pré-experimental referente ao envolvimento afetivo provavelmente interferiu com a emergência das relações condicionais que definem a formação de classes de estímulos equivalentes, uma vez que esses não formaram as classes de equivalência, mas formaram aquelas que envolviam outros estímulos que não tinham esse significado. $\mathrm{O}$ desempenho dos participantes no Teste de Equivalência Global, que envolvia todas as relações das quatro classes de equivalência testadas, esteve positivamente correlacionado com o relato verbal apresentado pelos participantes na Escala de Ciúme Romântico. Os resultados do teste das relações emergentes que envolviam o nome do namorado ou da namorada e o nome de uma mulher ou de um homem desconhecido (A1D1 e D1A1) também estão positivamente correlacionados com o resultado da Escala de Ciúme Romântico, o que apoia a hipótese de interferência formulada.

Esses resultados corroboram aqueles obtidos por Leslie et al. (1993), os quais concluíram que o efeito de interferência pode ocorrer devido ao conflito no controle de estímulos estabelecido na história pré-experimental e as contingências estabelecidas pelo experimentador. No estudo desses autores, foi demonstrado que sete de oito participantes classificados como ansiosos não formaram as classes de equivalência quando estímulos ameaçadores faziam parte das relações condicionais a serem estabelecidas: eles acertaram em média $30 \%, 40 \%$ e $50 \%$ diante das relações $\mathrm{C} 1 \mathrm{~A} 1, \mathrm{C} 2 \mathrm{~A} 2 \mathrm{e} \mathrm{C} 3 \mathrm{~A} 3$, respectivamente, enquanto os participantes não ansiosos acertaram 100\%, $95 \%$ e $95 \%$, respectivamente. Leslie et al. empregaram como estímulos expressões verbais com conotação ameaçadora (entrevista de emprego, falar em público, exame), enquanto no presente estudo, os estímulos em si não eram ameaçadores ou punitivos, mas a relação condicional emergente poderia ter esse tipo de função, eliciando respondentes que fazem parte do ciúme. Neste estudo, os participantes deviam, no teste das relações emergentes A1D1 e D1A1, relacionar o nome do namorado ou da namorada a um nome de mulher ou homem desconhecido, ou seja, apenas no teste de transitividade e de transitividade simétrica o efeito de interferência da história pré-experimental seria observado, enquanto que, no de Leslie et al., o efeito de interferência já podia ser observado na fase de treino. Os resultados desses dois estudos permitem sugerir, ainda, que estímulos pertencentes a classes de equivalência podem apresentar diferentes graus de relacionamento (related ness of equivalent stimuli; Bortoloti \& de Rose, 2009), isto é, demonstram que processos de significação previamente formados interferem na formação de classes de estímulos equivalentes.

No presente estudo verificou-se, ainda, que ao controle das contingências estabelecidas pelos experimentadores, foi adicionado o controle das características dos estímulos (topografia de controle de estímulos - Mcllvane, 1998), o que foi evidenciado pela diferença nas porcentagens de erros de respostas que requeriam relacionar figuras nos testes de linha de base e de simetria (BC e CB), em comparação com as que envolviam nomes e figuras ( $\mathrm{AB}, \mathrm{CD}, \mathrm{BA}, \mathrm{DC})$, com a única exceção foi das relações da Classe 4 (B4C4). Esse mesmo efeito foi observado nos testes de transitividade e de transitividade simétrica das relações $\mathrm{A} 3 \mathrm{D} 3$ e D3A3, bem como nos testes das relações C2A2, D2B2, D2A2; C3A3, D3B3, D3A3; C4A4, D4B4, D4A4. Esse fenômeno tem diferentes explicações na bibliografia sobre equivalência de estímulos, como aquelas baseadas na facilitação estabelecida pela possibilidade de nomear os estímulos (Arntzen \& Torunn, 2010), do efeito relativo ao significado (meaningfulness) dos estímulos (Fields et al., 2012) ou ainda da mediação de respostas verbais (Lowe \& Horne, 1996), além de se ter que considerar que as figuras poderiam ser mais difíceis de serem discriminadas uma das outras nas tentativas em que são sucessivamente ou simultaneamente apresentadas (cf., Saunders \& Green, 1999). Como o presente estudo não visou testar esse tipo de variável, não se fará uma discussão extensa sobre esse aspecto. Vale destacar, no entanto, que no caso das relações A1D1 e D1A1, que envolviam apenas nomes (dois estímulos nomeáveis em que um deles tem alto significado emocional) não se observou um efeito facilitador, não tendo havido desempenho melhor em comparação às relações que envolviam figuras não familiares. Essas relações são aquelas em que o participante teve que escolher o nome do namorado diante do nome de uma mulher desconhecida ou o nome da namorada diante do nome de um homem desconhecido, isto é, as relações consideradas como sendo as que caracterizam um conflito entre contingências. Portanto, o fato de os participantes terem apresentado porcentagens de respostas incorretas mais altas na formação das relações A1D1 e D1A1, em comparação às relações C1A1, D1B1, demonstra que, na formação da Classe 1, não houve efeito do controle de topografia de estímulos, sendo esse um dado adicional do efeito de interferência da história pré-experimental sobre o comportamento de escolha dos participantes.

Com relação ao desempenho dos participantes nos testes das relações A1D1 e D1A1, deve-se considerar, ainda, que elas envolvem um maior número de estímulos 
relacionados condicionalmente (dois nódulos) do que as relações A1C1, B1D1, C1A1, D1B1 (um nódulo). Diversos estudos demonstraram que essa é uma variável relevante na formação das classes de equivalência: relações que envolvem um maior número de nódulos (maior distância nodal) têm uma probabilidade menor de serem formadas (e.g., Fields, Landon-Jimenez, Buffington, \& Adams, 1995). Essa poderia ser a razão pela qual, nos testes em que o nome do namorado(a) deveria ser relacionado ao nome de mulher ou homem desconhecido (A1D1 e D1A1), o desempenho foi pior. Não obstante, o mesmo não foi observado diante das relações de transitividade A3D3 e A4D4, e de transitividade simétrica D2A2, D3A3, D4A4, que também são separadas por dois nódulos. Assim, como o efeito da distância nodal não foi observado na maioria das condições que envolvia dois nódulos em comparação àquelas que envolvia apenas um nódulo, não se pode atribuir a porcentagem maior de erros ao efeito da distância nodal no caso das relações A1D1 e D1A1. Esse dado é bastante interessante no sentido de fornecer evidência adicional à explicação formulada por Sidman (1994, ver também Saunders \& Green, 1999) de que a diferença no desempenho dos participantes decorre da história de reforço e não do grau com que os estímulos ficam relacionados devido à "distância associativa" (Fields et al., 1995).

Os participantes demoraram mais para emitir as respostas de escolha incorretas do que as corretas diante das relações transitivas e transitivas simétricas de todas as quatro classes. Esse dado está de acordo com a previsão feita a partir da bibliografia de que há uma covariação entre o desempenho em termos de precisão do responder (porcentagem de repostas corretas) e o tempo de reação (e.g., Dymond \& Rehfeldt, 2001). Uma análise complementar do efeito da história pré-experimental pode ser feita a partir desses dados, pois foi observado que o tempo de reação foi acentuadamente maior na emissão das repostas de escolha incorretas dos estímulos que envolviam o nome do namorado(a) - relações A1D1 e D1A1 - do que na emissão de respostas dessa classe que não envolviam esse nome e em comparação àquelas que estavam corretas. Essa diferença maior permite sugerir que, ao emitir as respostas de escolha em situação de conflito e com sentimento de ciúme, os participantes demoravam mais para responder. Por outro lado, se o participante não estivesse diante de contingências conflituosas, a familiaridade do estímulo (nome de pessoas, sendo uma delas conhecida), provavelmente, contribuído para respostas rápidas e corretas, conforme sugerem estudos que investigaram o efeito de variáveis com a familiaridade dos estímulos (Arntzen \& Torunn, 2010). Essa interpretação é especulativa e requer investigações com controle e isolamento da variável familiaridade dos estímulos. Por exemplo, mais uma das classes de estímulos equivalentes a ser formada poderia envolver relações entre estímulos familiares, para averiguar se, nesse caso, as classes teriam probabilidades diferentes de serem formadas. Outras possibilidades são a inclusão de fotos do namorado(a), ou ainda o nome do próprio participante em uma das classes a ser formada.
Durante a coleta de dados, algumas participantes afirmaram que não queriam escolher o nome do namorado diante do nome de outra mulher, mas o fizeram porque sabiam que essa era a reposta correta. Esses relatos não foram sistematicamente registrados, mas em estudos futuros, o registro desse tipo de comportamento, bem como de respostas fisiológicas eliciadas, por exemplo, respostas galvânicas da pele, como foi feito por Augustson, Dougher e Markham (2000), nas tentativas de escolha envolvendo a relação que apresentam conflito poderiam ser dados adicionais relevantes. Outro aspecto a ser considerado em estudos futuros é o tipo de amostra usada, uma vez que, no presente estudo, usou-se uma amostra, por conveniência, de estudantes universitários. Estudos futuros devem considerar a possibilidade de selecionar uma amostra de população clínica (como em Leslie et al., 1993 e Tyndall et al., 2004), com queixa de ciúme em níveis que interferem com o relacionamento amoroso.

De forma geral, pode-se concluir que a história pré-experimental referente ao envolvimento afetivo dos participantes interferiu na formação de classes equivalentes que eram formadas pelo nome do(a) namorado(a). Isso permite sugerir que o grau de relacionamento dos estímulos (Bortoloti \& de Rose, 2009) deve ser considerado ao se fazer análises funcionais de relações de equivalência, uma vez que pode ser constatado ter havido reações de conflito na escolha dos estímulos de comparação nos testes das relações emergentes e, consequentemente, diferentes graus de transferência de função.

\section{Referências}

Almeida, T., Rodrigues, K. R. B., \& Silva, A. A. (2008). O ciúme romântico e os relacionamentos amorosos heterossexuais contemporâneos. Estudos de Psicologia (Natal), 13, 83-90. doi:10.1590/S1413-294X2008000100010

Arntzen, E., \& Holth, P. (2000). Probability of stimulus equivalence as a function of class size $v s$. number of classes. The Psychological Record, 50, 79-104.

Arntzen, E., \& Torunn, L. (2010). Trained and derived relations with pictures versus abstract stimuli as nodes. The Psychological Record, 60, 659-678. Retrieved from http://opensiuc. lib.siu.edu/tpr/vol60/iss4/8

Augustson, E. M., Dougher, M. J., \& Markham, M. R. (2000). Emergence of conditional stimulus relations and transfer of respondent eliciting functions among compound stimuli. The Psychological Record, 50, 745-770.

Banaco, R. A. (2005, maio). Ciúme e inveja. Palestra proferida no I Congresso Brasileiro de Psicologia Clínica e da Saúde, Londrina, PR.

Barros, R. S., Galvão, O. F., Brino, A. L. F., Goulart, P. R. K., \& Mcllvane, W. J. (2005). Variáveis de procedimento na pesquisa sobre classes de equivalência: Contribuições para o estudo do comportamento simbólico. Revista Brasileira de Análise do Comportamento, 1, 15-27.

Bortoloti, R., \& de Rose, J. C. (2009). Assessment of the relatedness of equivalent stimuli through a semantic differential. The Psychological Record, 59, 563-590.

Capocio, V. P. (2008). Software Equivalência. Ribeirão Pires, SP.

Costa, N., \& Barros, R. S. (2010). Ciúme: Uma interpretação analítico-comportamental. Acta Comportamentalia, 18, 135-149. 
Haydu, V. B., Gaça, L. B., Cognetti, N. P., Costa, C. E. \& Tomanari, G. Y. (2015). Equivalência de Estímulos e Ciúme: Efeito de História Pré-Experimental.

Dack, C., McHugh, L., \& Reed, P. (2012). Transfer of judgments of control to a target stimulus and to novel stimuli through derived relations. Learning and Behavior, 40, 448-464. doi:10.3758/s13420-012-0066-6.

De Carvalho, M. P. (2009). Resistência à mudança de atitude preconceituosa racial avaliada pelo paradigma de equivalência de estímulos (Dissertação de mestrado em Psicologia, Universidade Federal de São Carlos, SP, Brasil).

Dixon, M. R., Refeldt, R. A., Zlomke, K. R., \& Robinson, A. (2006). Exploring the development and dismantling of equivalence classes involving terrorist stimuli. The Psychological Record, 56, 83-103.

Dube, W. V., \& Mcllvane, W. J. (1996). Some implications of a stimulus control topography analysis for emergent stimulus classes. In T. R. Zentall \& P. M. Smeets (Eds.), Stimulus class formation in humans and animals (pp. 197-218). Amsterdam, Netherlands: Elsevier.

Dymond, S., \& Rehfeldt, R. A. (2001). Supplemental measures and derived stimulus relations. Experimental Analysis of Human Behavior Bulletin, 19, 8-12. Retrieved from http://psy.swan.ac.uk/ staff/dymond/pubs/DymondRehfeldt.pdf

Dymond, S., Roche, B., Forsyth, J. P., Whelan, R., \& Rhoden, J. (2008). Derived avoidance learning: Transformation of avoidance response functions in accordance with same and opposite relational frames. The Psychological Record, 58, 269-286.

Fields, L., Arntzen, E., Nartey, R. K., \& Eilifsen, C. (2012). Effects of a meaningful, a discriminative, and a meaningless stimulus on equivalence class formation. Journal of the Experimental Analysis of Behavior, 97, 163-181. doi:10.1901/jeab.2012.97-163

Fields L., Landon-Jimenez, D. V., Buffington, D. M., \& Adams, B. J. (1995). Maintained nodal-distance effects in equivalence classes. Journal of the Experimental Analysis of Behavior, 64, 129-45. doi:10.1901/jeab.1995.64-129

Grey, I. M., \& Barnes, D. (1996) Stimulus equivalence and attitudes. The Psychological Record, 46, 243-270.

Lowe, C. F., \& Horne, P. J. (1996). Reflections on naming and other symbolic behavior. Journal of the Experimental Analy sis of Behavior, 65, 315-353. doi:10.1901/jeab.1996.65-315

Imam, A. A. (2006). Experimental control of nodality via equal presentations of conditional discriminations in different equivalence protocols under speed and no-speed conditions. Journal of the Experimental Analysis of Behavior, 85, 107 124. doi:10.1901/jeab.2006.58-04

Kato, O. M., de Rose, J. C., \& Faleiros, P. B. (2008). Topography of responses in conditional discrimination influences formation of equivalence classes. The Psychological Record, 58, 245-267.

Leslie, J. C., Ulster-Jordanstown, U., Tierney, K. J., Robinson, C. P., \& Keenan, M. (1993). Differences between clinically anxious and non-anxious subjects in a stimulus equivalence training task involving threat words. Psychological Record, 43, 153-161.

McIlvane, W. J. (1998). Teoria da coerência da topografia de controle de estímulos: Uma breve introdução. Temas em Psicologia, 6, 185-189.

McGlinchey, A., Fairhurst, C., \& Dillenburger, K. (2000) Accuracy of disclosure and contextual control in child abuse: Developing procedures within the stimulus equivalence paradigm. Behavior and Social Issues, 10, 1-17. doi:10.5210\%2Fbsi. v10i0.131

Medeiros, J. G., \& Nogueira, M. F. (2005). A nomeação de figuras como facilitadora do ler e escrever em crianças com dificuldades de aprendizagem. Psicologia: Teoria e Prática, 7, 107-126.
Moss-Lourenco, P., \& Fields, L. (2011). Nodal structure and stimulus relatedness in equivalence classes: Post-class formation preference tests. Journal of the Experimental Analysis of Behavior, 95, 343-368. doi:10.1901/jeab.2011.95-343

Neves, S. M., Vandenberghe, L. M. A., Oliveira, L. H. R., Silva, A. V., de Oliveira, K. C. F., Oliveira, J. Di S., ... Villane, M. C. S. (1999). O modelo da equivalência de estímulos na análise de distúrbios de ansiedade: Os efeitos da história experimental e da qualidade dos estímulos em sujeitos ansiosos e não ansiosos. Revista Brasileira de Terapia Comportamental e Cognitiva, 1, 57-66.

Plaud, J. J. (1995). The formation of stimulus equivalence classes: Fear-relevant and fear-irrelevant stimulus classes. The Psychological Record, 45, 207-222.

Plaud, J. J., Gaither, G. A., Franklin, M., Weller, L. A., \& Barth, J. (1998). The effects of sexually explicit words on the formation of stimulus equivalence classes. The Psychological Record, 48, 63-79.

Ramos, A. L. M., Yazawa, S. A. K., \& Salazar, A. F. (1994). Desenvolvimento de uma escala de ciúme romântico. Psicologia: Teoria e Pesquisa, 10, 439-451.

Saunders, R. R., \& Green, G. (1999). A discrimination analysis of training-structure effects on stimulus equivalence outcomes. Journal of the Experimental Analysis of Behavior, 72, 117137. doi:10.1901/jeab.1999.72-117

Sidman, M. (1971). Reading and auditory-visual equivalence. Journal of Speech and Hearing Research, 14, 5-13.

Sidman, M. (1986). Functional analysis of emergent verbal classes. In T. Thompson \& M. D. Zeiler (Eds.), Analysis and integration of behavioral units (pp. 213-245). Hillsdale, NJ: Erlbaum.

Sidman, M. (1994). Equivalence relations and behavior: A research story. Boston, MA: Authors Cooperative.

Sidman, M. (2000). Equivalence relations and the reinforcement contingency. Journal of the Experimental Analysis of Behavior, 74, 127-146. doi:10.1901/jeab.2000.74-127

Sidman, M., Kirk, B., \& Willson-Morris, M. (1985). Six-member stimulus classes generated by conditional-discrimination procedures. Journal of the Experimental Analysis of Behavior, 43, 21-42. doi:10.1901/jeab.1985.43-21

Sidman, M., \& Tailby, W. (1982). Conditional discrimination vs. matching to sample: An expansion of the testing paradigm. Journal of the Experimental Analysis of Behavior, 37, 5-22. doi:10.1901/jeab.1982.37-5

Skinner, B. F. (1976). Walden two. Englewood Cliffs, NJ: Prentice Hall. (Original publicado em 1948)

Tyndall, I. T., Roche, B., \& James, J. E. (2004). The relation between stimulus function and equivalence class formation. Journal of the Experimental Analysis of Behavior 81, 257-266. doi:10.1901/jeab.2004.81-257

Watt, A., Keenan, M., Barnes, D., \& Cairns, E. (1991). Social categorization and stimulus equivalence. The Psychological Record, 41, 33-50. 